This is the peer reviewed version of the following article: [Ohara M, Tomoda F, Koike T, Liu H, Uno K, Nitta A, Inoue H. Pubertal administration of antiserum against nerve growth factor regresses renal vascular remodeling in spontaneously hypertensive rats. Clin. Exp. Pharmacol. Physiol. 2015; 42: 687-94], which has been published in final form at [10.1111/1440-1681.12411]. This article may be used for non-commercial purposes in accordance with Wiley Terms and Conditions for Self-Archiving.

\title{
PUBERTAL ADMINISTRATION OF ANTISERUM AGAINST NERVE \\ GROWTH FACTOR REGRESSES RENAL VASCULAR REMODELING IN
}

\section{SPONTANEOUSLY HYPERTENSIVE RATS}

Maiko Ohara M.D. ${ }^{1}$, Fumihiro Tomoda M.D., Ph.D. ${ }^{1}$, Tsutomu Koike M.D., Ph.D. ${ }^{1}$,

Hexing Liu M.D., Ph.D. ${ }^{1}$, Kyosuke Uno Ph.D. ${ }^{2}$, Atsumi Nitta Ph.D. ${ }^{2}$ and Hiroshi Inoue M.D., Ph.D ${ }^{1}$.

(1) The Second Department of Internal Medicine and (2) the Department of Pharmaceutical Therapy \& Neuropharmacology, University of Toyama, Toyama, Japan.

Running title: NGF and renal vascular remodeling in SHR

Address for correspondence:

Fumihiro Tomoda, M.D., Ph.D.

The Second Department of Internal Medicine, University of Toyama,

2630 Sugitani, Toyama, 930-0194, Japan

Telephone: +81-76-434-7297

Fax: +81-76-434-5026

E-mail: tomoda@med.u-toyama.ac.jp 


\section{Abstract}

To investigate the role of nerve growth factor (NGF) in the development of hypertensive renal vascular remodeling, antiserum against NGF (anti-NGF) or vehicle was injected at 3 weeks of age in spontaneously hypertensive rats (SHR) and Wistar-Kyoto (WKY) rats ( $n=9$ for each treatment in each strain). Flow-pressure (F-P) and pressure-glomerular filtration rate (P-GFR) relationships at vasodilated perfused kidneys were determined at 10 weeks of age. In the vehicle rats, blood pressure, renal noradrenaline content, the gradient of F-P (minimal vascular resistance at pre- and post-glomerular vasculature) and the X-intercept of P-GFR (preglomerular : postglomerular vascular resistance ratio) were greater in SHR than in WKY rats, although the gradient of P-GFR (glomerular filtration capacity) did not differ significantly between the strains. Blood pressure and renal noradrenaline content were lower in SHR receiving anti-NGF than in SHR receiving vehicle, although such difference was not observed in WKY rats. The gradient of F-P was less but the gradient of P-GFR was greater in SHR receiving anti-NGF compared with SHR receiving vehicle, although the similar differences did not occur in WKY rats. Blood pressure and renal noradrenaline content remained greater in SHR treated with anti-NGF compared with WKY rats treated with vehicle; however, the gradient of F-P did not differ significantly between them. Contrary, anti-NGF did not 
affect the X-intercept of P-GFR in either strain. In conclusion, NGF could contribute to the genesis of renal vascular remodeling, at least in part, through modification of renal sympathetic activity and blood pressure in SHR.

Key words: nerve growth factor, immunosympathectomy, renal vascular remodeling, spontaneously hypertensive rat, vasodilated perfused kidney 


\section{Introduction}

In systemic hypertension, it is well known that resistance vessels become thicker or encroach into the lumen in kidneys as well as in other organs $[1,2]$. In spontaneously hypertensive rats (SHR), a model of human essential hypertension, such renal vascular changes occur mainly in the preglomerular vessels at the prehypertensive or early hypertensive stage [3-8]. This structural remodeling of the renal vasculature can promote hypertension by increasing renal vascular resistance and reducing both glomerular filtration and sodium excretion in SHR [8]. Because the sympathetic nervous system controls vascular growth and structures [9, 10] and its activity is enhanced in SHR [11, 12], the increase in renal sympathetic nerve activity is related with the genesis of renal vascular remodeling in SHR [7].

Nerve growth factor (NGF) is a protein required for development and maintenance of the sympathetic neurons [13, 14]. NGF is synthesized in organs innervated by sympathetic nerves, incorporated by sympathetic nerve endings and transported through axons to neural cell bodies [13, 14]. In most tissues including kidneys, tissue levels of NGF protein correlate closely with the density of sympathetic innervations and sympathetic nerve activity [15-17]. Therefore, in hypertension, renal synthesis of NGF can modulate renal sympathetic neural activity, thereby affecting both 
the renal vasculature and blood pressure. This thesis is supported by the observation that NGF mRNA and protein increased in kidneys as well as mesenteric arteries of SHR [15, 18-20]. Furthermore, an administration of antiserum against NGF (anti-NGF) attenuated or prevented the development of vascular hypertrophy and hypertension concomitantly with reducing sympathetic neural innervation and activity in the prehypertensive SHR [21-25]. Conversely, NGF treatment of normotensive Wistar-Kyoto (WKY) rats induced vascular hyperinnervation and increased vascular wall thickness, which are characteristics of SHR [26]. However, it is still unknown how an overproduction of NGF can lead to vascular remodeling via its influences on sympathetic neural innervation and activity in the kidneys of SHR.

In the present study, therefore, the following experiments were performed to explore that higher level of renal NGF would cause renal vascular remodeling and high blood pressure via its effect on renal sympathetic nerve activity in SHR using WKY rats as a normotensive control. Firstly, the capability of NGF synthesis was compared between the two strains in several organs at 3 weeks of age. Secondly, in both strains, renal vascular structures and blood pressure as well as renal sympathetic neural activity were investigated following the immunosympathectomy using anti-NGF at the pubertal stage. 


\section{Results}

Experiment 1: Expression of NGF mRNA in organs of 3-week-old SHR and WKY

rats

Expression of NGF mRNA was significantly elevated in the kidney, left ventricle (not in the right ventricle) and submandibular glands of SHR compared with those of WKY rats at 3 weeks of age (Table 1). Especially, renal expression of NGF mRNA was 3.9 times higher in SHR than in WKY rats.

Experiment 2: Effects of immunosympathectomy on the renal vasculature, renal catecholamine content and blood pressure

In the rats treated with vehicle, systolic blood pressure was significantly higher in SHR than in WKY rats $(P<0.001)$ at 9 weeks of age, although pulse rate did not differ significantly between the two strains $(P=0.68$, Table 2$)$. At 10 weeks of age, renal noradrenaline content, left ventricular weight and right kidney wet weight were greater in SHR than in WKY rats $(P<0.001, P<0.001$ and $P=0.04$, respectively), whereas body weight was slightly less in SHR than in WKY rats $(P=0.011)$.

Systolic blood pressure, pulse rate and renal noradrenaline content did not differ significantly between WKY rats treated with anti-NGF and with vehicle $(P=0.12$, 0.081 and 0.25 , respectively). Contrary, systolic blood pressure and renal noradrenaline 
content were less in SHR treated with anti-NGF than in SHR treated with vehicle $(P=$ 0.018 and 0.006 , respectively), although pulse rate did not differ significantly between the two groups $(P=0.78)$. However, the effects of anti-NGF on systolic blood pressure and renal noradrenaline content did not differ significantly between the two strains $(P=$ 0.40 and 0.11 for strain $\times$ treatment interaction, respectively). Additionally, both parameters remained greater in SHR treated with anti-NGF compared with WKY rats treated with vehicle ( $P<0.001$ for each).

There was no significant difference in body weight, pulse rate and organ weights between the rats treated with anti-NGF and with vehicle in either strain.

\section{Haemodynamic findings in vasodilated kidneys at 10 weeks of age}

A linear relationship was seen between perfusion flow and arterial distending pressure within each individual perfusion experiment, with Pearson correlation coefficients $\left(\mathrm{r}^{2}\right)$ ranging from 0.96 to 0.99 (Figure1). A linear relationship was also seen between arterial distending pressure and GFR within each individual experiment, with $\mathrm{r}^{2}$ ranging from 0.96 to 0.99 (Figure 2).

In the rats treated with vehicle, the gradient of the flow-pressure relationship was significantly greater in SHR than in WKY rats at 10 weeks of age $(P<0.001$, Table 3). In contrast, the $\mathrm{X}$ intercept of the pressure-GFR relationship was significantly higher 
in SHR than in WKY rats $(P<0.001)$, although the gradient of the pressure-GFR relationship did not differ significantly between the two strains $(P=0.15)$. These results indicate that minimal vascular resistance was elevated with an increase in preglomerular : postglomerular vascular resistance ratio in the kidneys of SHR, although glomerular filtration capacity was normal.

The gradient of the flow-pressure relationship, and the $\mathrm{X}$ intercept and the gradient of the pressure-GFR relationship did not differ significantly between WKY rats treated with anti-NGF and with vehicle (Table 3, $P=0.19,0.28$ and 0.30 , respectively). Conversely, the gradient of the flow-pressure relationship was less in SHR treated with anti-NGF than in SHR treated with vehicle (Table 3, $P<0.001$ ), although the $\mathrm{X}$ intercept of the pressure-GFR relationship did not differ significantly between the two groups (Table 3, $P=0.23$ ). The gradient of pressure-GFR relationships was also greater in SHR treated with anti-NGF than in SHR treated with vehicle (Table $3, P=0.003$ ). Additionally, the effects of anti-NGF on minimal renal vascular change and glomerular filtration capacity were significantly different between the two strains ( $P=0.009$ and 0.036 for strain $\times$ treatment interaction, respectively). As a result, there were no significant differences in minimal vascular resistance between SHR treated with anti-NGF and WKY rats treated with vehicle $(P=0.47)$, whereas glomerular filtration 
capacity was greater in SHR treated with anti-NGF than in WKY rats treated with vehicle $(P=0.004)$.

\section{Histological findings in the vasodilated kidneys at 10 weeks of age}

In the rats treated with vehicle, the internal luminal area of the interlobular arteries (one of the preglomerular resistance vessels) was significantly smaller in SHR than in WKY rats at 10 weeks of age $(P=0.010)$ (Figure 3 , Table 4). The wall cross-sectional area of these vessels was significantly greater in SHR than in WKY rats $(P<0.001)$. As a result, the wall : lumen ratio was significantly greater in SHR compared with WKY rats $(P<0.001)$. Glomerular tuft area was also greater in SHR than in WKY rats at both superficial and juxtamedullary nephrons ( $P<0.001$ for each). There were no increases in mesangial matrix and no glomerular sclerosis in either strain.

The internal luminal area and wall cross-sectional area of the interlobular arteries did not differ significantly between WKY rats treated with anti-NGF and with vehicle ( $P=0.22$ and 0.97 , respectively) (Figure 3, Table 4). Contrary, the internal luminal area was greater but the wall cross-sectional area was less in SHR treated with anti-NGF compared with SHR treated with vehicle ( $P=0.001$ and 0.033 , respectively). As a result, the wall : lumen ratio of the interlobular arteries was less in SHR treated with anti-NGF than in SHR treated with vehicle $(P<0.001)$, although it did not differ 
significantly between the two treatment groups in WKY rats $(P=0.23)$. Additionally, the effects of anti-NGF on internal luminal area and wall : lumen ratio were significantly different between both strains ( $P<0.001$ in each for strain $\times$ treatment interaction). These two parameters also did not differ significantly between SHR treated with anti-NGF and WKY rats treated with vehicle ( $P=0.12$ and 0.19 , respectively) (Table 4). On the other hand, anti-NGF did not affect glomerular tuft area and induce any morphological changes suggestive of glomerular damages in either strain.

\section{Discussion}

To the best of our knowledge, the present study was the first to ascertain the effects of immunosympathectomy using anti-NGF on renal vascular structural changes in hypertensive animals. The major findings of the present study are as follows. First, renal expression of NGF mRNA was enhanced in SHR compared with WKY rats at the pubertal period, i.e., 3 weeks of age. In the kidneys of young SHR aged 10 weeks, vascular narrowing occurred predominantly at the preglomerular resistance vessels with vascular hypertrophy, a finding consistent with hypertrophic inward remodeling, despite there being no abnormality in the whole kidney filtration capacity. These findings in SHR are consistent with those in the previous reports [4-8]. Secondly, in SHR, the single administration of anti-NGF at 3 weeks of age attenuated hypertension as well as 
elevation in renal noradrenaline content 7 weeks later, but did not prevent these completely. Conversely, the anti-NGF treatment did not affect blood pressure and renal noradrenaline content in WKY rats. Thirdly, most importantly, the development of renal vascular structural alterations was affected by the anti-NGF treatment at the pubertal stage in SHR. That is, in the kidneys of SHR, the anti-NGF treatment prevented the development of vascular narrowing in the whole renal resistance vessels (i.e., pre- and post-glomerular vasculature), although it did not influence the preglomerular : postglomerular vascular resistance ratio. The anti-NGF treatment also enhanced glomerular filtration capacity against pressure in SHR. Furthermore, the influences of anti-NGF treatment on renal vascular structures were specific for SHR, because the anti-NGF treatment did not affect any parameters for renovascular structures in WKY rats.

The possible mechanisms of renal structural responses to the anti-NGF treatment

\section{in SHR}

It is well-known that an overactivation of sympathetic nervous system occurs prior to the onset of hypertension [11, 12]. Because the sympathetic nervous system exerts a hypertrophic effect on the cardiovascular structures [9, 10], enhanced sympathetic nerve activity or sympathetic hyperinnervation can induce cardiovascular 
structural remodeling such as vascular or cardiac hypertrophy [2, 10, 12]. Such cardiovascular structural remodeling can amplify responses of the vessels and heart to vasoconstrictor stimuli, leading to increases in total peripheral resistance and cardiac output $[1,2]$. Therefore, cardiovascular structural remodeling secondary to increased sympathetic neural activity has long been regarded as one of the factors to promote and maintain hypertension $[1,2,8,10]$. Furthermore, vascular overproduction of NGF might be responsible for the enhanced vascular sympathetic neural activity that is implicated in the vascular remodeling of hypertensive animals [12, 15, 21-24]. However, in SHR, the relationships between NGF and cardiovascular structures were evaluated mainly in the mesenteric arteries, heart and hindquarter vascular beds [21-25], but not in renal vessels and glomeruli.

In the present study, the anti-NGF treatment inhibited the development of vascular narrowing at the whole renal resistance vessels in the kidneys of SHR and this vascular widening appears to have occurred in the preglomerular and postglomerular resistance vessels to a similar degree. This renal haemodynamic alteration was characterised histologically by the prevention of the development of hypertrophic inward remodeling at the interlobular arteries in the kidneys of SHR. The anti-NGF treatment also enhanced glomerular filtration against pressure without any 
morphological changes in glomeruli in the kidneys of SHR. Thus, in SHR, the single administration of anti-NGF at the pubertal period regressed the renal vascular remodeling, although it did not normalise blood pressure and renal noradrenaline content. However, the previous reports demonstrated that anti-NGF alone could not prevent the development of cardiac hypertrophy and vascular remodeling in mesenteric arteries and hindquarter vascular beds in SHR [23-25]. Accordingly, in SHR, renal vasculature appeared to exhibit hyper-responsiveness to the anti-NGF treatment compared with heart and other vascular beds. Indeed, in the present study, anti-NGF did not reduce left ventricular weight in SHR. Although it seems hard to explain the differences of responsiveness to anti-NGF between renal vasculature and other vascular beds, renal vasculature might be highly sensitive to the inhibition of sympathetic neural activity induced by anti-NGF in SHR. Alternatively, because NGF has also direct proliferative actions on vascular smooth muscle cells, endothelial cells and inflammatory cells [27-29], the renal vascular structures might be strongly affected by the additive inhibition of anti-NGF on the growth of these non-neuronal cells in SHR. Of course, reduced systemic pressure and/or inhibited renin angiotensin aldosterone system secondary to sympathetic inhibition should play important roles in the regression of renal vascular remodeling following the anti-NGF treatment in SHR [30, 31]. 


\section{The influences of the anti-NGF treatment on blood pressure and renal} noradrenaline content in SHR

NGF synthesis was enhanced in the organs of SHR compared with those in WKY rats at the pubertal stage. This phenomenon is supported by the previous reports demonstrating that in SHR, cardiac content of NGF peptide is higher at 18-24 days [18] and renal NGF m-RNA is enhanced at 3 and 10 weeks of age [20]. The difference of NGF synthesis in organs between the two strains seems to be due to the genetic differences in NGF gene or NGF gene transcription, but not in physiological influence of blood pressure, because it occurred prior to the onset of hypertension [20] .

In the present study, the single administration of anti-NGF was performed for immunosympathectomy at 3 weeks of age at which the enhanced synthesis of NGF occurred in organs of SHR. However, the sympathectomy using anti-NGF alone was not so powerful to normalise renal noradrenaline content in SHR. The similar incomplete sympathectomy was reported previously in SHR treated with anti-NGF alone [23-25]. Because the immunosympathectomy using anti-NGF alone spares the catecholamine-producing cells of the adrenal medulla, reflex-induced secretion of catecholamines from adrenal glands might occur in response to the removal of sympathetic neural support $[10,23]$. Alternatively, re-innervation to the kidney might 
occur following the sympathectomy in SHR [10, 23]. These two phenomena secondary to the immunosympathectomy using anti-NGF alone could reduce the efficacy of immunosympathectomy on renal sympathetic neural activity. Furthermore, anti-NGF might also affect baroreflex function in the central nervous system to alter sympathetic outflow to the kidneys [32], because neurotrophic factors including NGF and their receptors are highly expressed in the brain stem [13, 33]. Indeed, an injection of brain derived neurotrophic factor (one of the neurotrophic factors) into the nucleus tractus solitaries, the area critical for baroreflex function, can increase arterial pressure in rats [34]. Therefore, the inhibitory effects of anti-NGF on renal sympathetic nerve activity could be mediated by the influences of anti-NGF on pre-ganglionic neurons as well as post-ganglionic neurons.

\section{Implications of renal structural alterations following the anti-NGF treatment in}

\section{SHR}

Taking into account the amplification of responses to vasoactive stimuli in vascular remodeling $[1,2,30,31]$, the following sequence of events may ensue in the kidney of SHR. Hypertrophic inward remodeling occurred in the preglomerular resistance vessels without changes in glomerular filtration capability in the kidneys of young SHR. These vascular structural changes could enhance renal vasoconstriction 
predominantly in the preglomerular vessels and reduce glomerular filtration, thereby decreasing renal excretory capability, even if the neurohumoral influences on the kidneys remain to be unchanged [1, 2, 8]. Consequently, systemic arterial pressure would be expected to increase in a way that offsets the reduced renal excretory function.

The anti-NGF treatment significantly affected the above mentioned changes in the renal vascular tree in SHR. That is, anti-NGF dilated averaged vascular lumen at the whole renal resistance vessels and enhanced glomerular filtration capability against pressure. These effects of anti-NGF on renal vascular remodeling could reduce the amplifier characteristics of vessels in the kidneys of SHR, thereby improving the reduction in renal excretory function $[1,2,8]$. However, theses expected effects of anti-NGF on renal excretory function could be depressed possibly by the residual elevation in preglomerular : postglomerular vascular resistance ratio in the renal resistance vessels of SHR. Therefore, the total effects of anti-NGF on renal vasculature could not be sufficient enough to normalise renal excretory function in SHR. Of course, to confirm this hypothesis, further studies are needed to explore the changes in other factors, including vascular reactivity and endothelial function, that could confound and modify such intrarenal haemodynamics [30, 31]. Actually, in SHR treated with anti-NGF, the residual elevation in renal sympathetic neural activity could affect the 
renal pressure-natriuresis relationship, thereby disturbing the normalisation of renal excretory function [32].

\section{Methodological considerations}

The methodological aspects of the present study deserve comments. First, single administration of anti-NGF at the pubertal period was used to perform immunosympathectomy in SHR. Brock et al demonstrated in SHR, that an intraperitoneal injection of a single dose (33 $\mu \mathrm{L} \cdot \mathrm{g}$ of body weight $\left.{ }^{-1}\right)$ of antiserum to NGF on the 19 or 24 postnatal day inhibited the later developments of hypertension as well as increase in sympathetic neural activity at 12 to 15 weeks of age [21]. In the present study, therefore, we specifically selected the similar route, dosage and age of the treatment with anti-NGF as proposed by Brock [21], although several reports did not support the effectiveness of anti-NGF alone on immunosympathectomy [23-25]. Second, renal vascular structure was assessed haemodynamically using an in vitro, maximally dilated whole-kidney perfusion technique, as in previous studies [6, 7, 35]. In contrast with histological analysis, this physiological technique is accurate and sensitive enough to detect subtle changes in lumen diameter in resistance vessels, as follows [2, 8]. It can determine vascular luminal changes at the whole renal resistance vessels (i.e., pre- and post-glomerular vasculature). It can also determine whether the luminal changes are 
confined to either the pre- or post-glomerular circulation. However, we cannot determine whether luminal changes at the preglomerular circulation occurred in the afferent arteriole, larger upstream vessels or both, or whether luminal changes were located in the cortical or medullary vessels. Third, histological evaluation of tyrosine hydroxylase (a rate-limiting enzyme to synthesise cathecolamines), histological staining of growth-associated factor 43 and neurofilament (markers for sympathetic innervation) or renal noradrenaline spillover could be suitable for the precise evaluations of renal sympathetic innervation and activity and support the present data concerning renal catecholamine content $[12,24,36-38]$. Fourth, anti-NGF treatment seemed to affect both afferent and efferent renal nerve, because NGF regulates innervation and neural activity in both nerves [13, 33]. However, it could not be elucidated in the present study whether anti-NGF affected either efferent, afferent or both. Fifth, most importantly, the cause and effect relationship between overproduction of renal NGF and vascular remodeling could not be deduced only from the present data. It is needed for the definite conclusion on this regard to examine whether the administration of NGF to WKY rats at 3 weeks of age can induce the increase in sympathetic innervation and vascular remodeling characteristic to the kidneys of SHR.

Although the study was limited for these reasons, it clearly demonstrated that 
anti-NGF prevented the later development of vascular narrowing in the whole renal resistance vessels without normalisation of blood pressure and renal sympathetic activity in SHR. These results suggest that in the kidneys of SHR, NGF could be associated with the genesis of vascular remodeling, partially mediated by its modification of sympathetic activity and blood pressure.

\section{Methods}

\section{Animals}

The study design and experimental protocols were in accordance with our institutional guidelines for animal research and the present study was approved by the Animal Experiment Committee of the University of Toyama. Three-week-old male SHR and WKY rats from the Izumo strain were purchased ( $\mathrm{n}=24$ in each strain). All rats were fed on a normal salt $(0.3 \% \mathrm{NaCl})$ diet and housed in a room maintained at constant temperature $\left(23-25^{\circ} \mathrm{C}\right)$ with a 12-hour light/dark cycle throughout the study period.

\section{Experiment 1: NGF mRNA levels in organs of 3-week-old SHR and WKY rats}

In three-week-old SHR and WKY rats ( $\mathrm{n}=6$ in each strain), kidneys, heart and submandibular glands were perfused by diethyl pyrocarbonate (DEPC) under anaesthesia and then removed. The extract of total RNA was extracted by homogenisation of the removed organs with TRIsure (Bioline, London, UK) according 
to the manufacture's instruction. The extracts of total RNA (300 ng) was mixed with 25 pmol oligo (dT) primer, 50 pmol random 6 mers, Prime Script ${ }^{\circledR}$ Buffer and Prime Script $^{\circledR}$ RT Enzyme Mix (TaKaRa, Shiga, Japan) and subsequently cDNA was synthesised by reverse transcription polymerase chain reaction (RT-PCR). The reverse transcription was run at $37^{\circ} \mathrm{C}$ for $30 \mathrm{~min}$. Real-time PCR was performed with Power SYBR $^{\circledR}$ Green PCR Master Mix, using a TaKaRa Dice Real Time System TP800 (TaKaRa, Shiga, Japan). The replication was carried out with the following cycling protocol (5 min of heat activation of the enzyme at $95^{\circ} \mathrm{C}, 45$ cycles of denaturation at $95^{\circ} \mathrm{C}$ for $20 \mathrm{~s}$, annealing at $59{ }^{\circ} \mathrm{C}$ for $20 \mathrm{~s}$, and extension at $72{ }^{\circ} \mathrm{C}$ for $20 \mathrm{~s}$ ). Primer sequences used for PCR were as follows: $5 `$ - ACCTCTTCGGACACTCTGGA -3`and 5`- GTCCGTGGCTGTGGTCTTAT -3` as rat NGF primers (up and down) and 5`CTCAGTGCCTCACTCCATCA-3` and 5`-CTTCCTTTGCTTCGACCTTG-3` as rat 36B4 primers (up and down). Finally, the relative amount of each transcript was normalised by the expression of ribosomal protein, large, P0 (36B4) and expressed using $\Delta \Delta \mathrm{Ct}$ method [39, 40].

Experiment 2: Effects of immunosympathectomy on the renal vasculature, renal catecholamine content and blood pressure 
According to the method of immunosympathectomy proposed by Brock et al [21], the animals were randomly assigned to receive an intraperitoneal injection with a single dose (40 $\mu \mathrm{L} \cdot \mathrm{g}$ of body weight ${ }^{-1}$ ) of either rabbit antiserum against the $\beta$-subunit of NGF (anti-NGF; Cedarlane Laboratories Limited, Hornby, Ontario, Canada) or rabbit serum at 3 weeks of age ( $n=9$ of each treatment in each strain). At 9 weeks of age, systolic blood pressure was measured in the conscious state by the tail-cuff method. Prior to the measurement of blood pressure, rats had been trained to become accustomed to the equipment. At 10 weeks of age, each rat was subjected to the following experiments using perfused kidneys.

\section{In vitro measurements in maximally vasodilated, perfused kidneys}

The experimental procedure has been reported elsewhere in detail [6, 7, 35]. In brief, after the rat was anaesthetised with pentobarbitone sodium, the right kidney was removed to measure renal noradrenaline content. Then, the abdominal aorta was isolated $1 \mathrm{~cm}$ proximally and distally to the left renal artery. All visible branches from the isolated aorta were ligated excepting the left renal artery and the mesenteric artery. The left ureter and the mesenteric artery were cannulated to allow urine collection and measurement of aortic pressure close to the left renal artery (i.e., renal arterial inflow pressure), respectively. A catheter connected to the perfusion set up was inserted in the 
retrograde fashion through the abdominal aorta to a position distal to the left kidney. The aorta was tied off just above the mesenteric artery to make a closed arterial circuit including the left kidney, and the left renal vein was cut. Thereafter, perfusion of the left kidney was commenced with oxygenated Tyrode's solution supplemented with $20 \mathrm{~g} \cdot \mathrm{L}^{-1}$ dextran, $0.9 \mathrm{mmol} \cdot \mathrm{L}^{-1}$ sodium nitroprusside, $10 \mathrm{mg} \cdot \mathrm{L}^{-1}$ furosemide and $1 \mathrm{~g} \cdot \mathrm{L}^{-1}$ inulin. The rat was killed with overdose of pentobarbital sodium, and the renal capsule was removed. A needle (25 gauge, inner diameter $0.3 \mathrm{~mm}$ ) was inserted perpendicular into the renal cortex for intrarenal tissue pressure measurement.

Thirty minutes after initiating renal perfusion, collection of urine and measurements of arterial inflow pressure and intrarenal tissue pressure were repeated during 7 or 8 stepwise increments of perfusion flow from 2 to $9 \mathrm{~mL} / \mathrm{min}$. Urinary volumes were determined gravimetrically. Inulin concentrations in the perfusate and urine were measured, and glomerular filtration rate (GFR) was calculated as inulin clearance. After the final measurements, the perfusate was changed to a fixative containing $2 \% \mathrm{w} / \mathrm{v}$ formaldehyde and $0.5 \% \mathrm{w} / \mathrm{v}$ glutaraldehyde in $0.075 \mathrm{~mol} \cdot \mathrm{L}^{-1}$ phosphate buffer $(\mathrm{pH}=7.2)$, with which the left kidney was perfused at a rate of 3 $\mathrm{mL} \cdot \mathrm{min}^{-1}$ for $60 \mathrm{~min}$. The perfusion of the fixative did not alter the perfusion pressure in this preparation, suggesting that there was no effect on the relaxed status of the 
vasculature. The left kidney was then removed and placed in $7.4 \% \mathrm{w} / \mathrm{v}$ formaldehyde. The kidney was sliced into $2 \mathrm{~mm}$ thickness and every second slice was embedded in paraffin. One section from each block was cut at $2 \mu \mathrm{m}$ thickness and stained with periodic acid-Schiff (PAS). At the end of experiment, the heart was dissected so that the left and right ventricle was weighed separately.

\section{Renal haemodynamics and histological appearance in the perfused kidneys}

In the maximally vasodilated, perfused kidneys, neurohumoral and active autoregulatory control systems are assumed to be inoperative; therefore, the analysis of haemodynamic behavior enables us to perform the following functional assessments of structural properties in the renal resistance vessels $[6,7,35]$. In the flow-pressure relationship, the gradient is the renal vascular resistance at maximal dilatation (i.e., minimal renal vascular resistance), which is an accurate index of averaged overall lumen dimensions of the preglomerular and postglomerular vasculature. In the pressure-GFR relationship, the intercept of the line with the pressure axis (i.e., the threshold pressure for commencing filtration) and the gradient of the line reflect preglomerular : postglomerular vascular resistance ratio and glomerular filtration capacity, respectively.

To estimate the above-mentioned 3 functional parameters, the flow-pressure 
and pressure-GFR relationships were established by the use of the perfusion flow, arterial distending pressure and GFR at each flow rate in the present study. In the relationships, arterial distending pressure (i.e., transmural pressure), calculated as arterial inflow pressure minus renal tissue pressure, was adopted as pressure, because tissue pressure built up during the perfusion, thereby inhibiting distension of the vasculature $[6,7,35]$.

In renal morphological analysis, cross-sectional areas of the wall and lumen in the proximal interlobular arteries and glomerular tuft area were measured with a digitising tablet (SD-510C, Wacom Co) under light microscopy. The wall-to-lumen ratio (i.e., media : lumen ratio) was calculated by dividing the cross-sectional area of the wall by that of the lumen. The histological appearances of glomeruli were also evaluated. The measurements were performed randomly in 10 to 15 vessels, 30 to 40 superficial glomeruli and 20 to 30 juxtamedullary glomeruli per animal.

\section{Renal noradrenaline content}

Renal noradrenaline content was measured in the right kidney removed at the renal perfusion experiments, as follows [7]. The kidneys were homogenised with $2 \mathrm{~mL}$ of $0.4 \%$ perchloric acid containing $0.1 \% \mathrm{w} / \mathrm{v}$ EDTA at $0^{\circ} \mathrm{C}$. The homogenates were centrifuged at 6000 r.p.m for 15 min. The noradrenaline levels of supernatant fluids 
were measured by high-performance liquid chromatography and electrochemical detection.

\section{Statistical analysis}

All data are expressed as mean \pm SEM. Inflow volume and GFR are expressed as values divided by wet weight of the right kidney. The assumption that flow-pressure and pressure-GFR relationships were linear in all individual experiments was tested using the Pearson correlation coefficient in simple regression analysis. The slope and X intercept of these relationships were derived from the individual regression lines. Differences between the strains and differences between the treatments were determined using one-way analysis of variance (ANOVA). Comparisons of the effect of anti-NGF treatment between the 2 strains were tested by two-way ANOVA. Two-tailed $P<0.05$ was considered significant.

Disclosure: The authors declared no conflict of interest.

\section{References}

1. Mulvany MJ. Small artery remodelling in hypertension. Basic Clin Pharmacol Toxicol. 2012; 110: 49-55.

2. Wright CE, Angus JA, Korner PI. The interactive vascular resistance amplifier and non-interactive reviewers. J Hypertens. 2002; 20: 1023-1027. 
3. Nørrelund H, Christensen KL, Samani NJ, Kimber P, Mulvany MJ, Korsgaard N. Early narrowed afferent arteriole is a contributor to the development of hypertension. Hypertension. 1994; 24: 301-308.

4. Kett MM, Alcorn D, Bertram JF, Anderson WP. Enalapril does not prevent renal arterial hypertrophy in spontaneously hypertensive rats. Hypertension.1995; 25: 335-342.

5. Skov K, Mulvany MJ, Korsgaard N. Morphology of renal afferent arterioles in spontaneously hypertensive rats. Hypertension.1992; 20: 821-827.

6. Kinuno H, Tomoda F, Koike T, Takata M, Inoue H. Effects of uninephrectomy on renal structural properties in spontaneously hypertensive rats. Clin Exp Pharmacology Physiol. 2005; 32: 173-178.

7. Tomoda F, Bergström G, Evans RG, Anderson WP. Evidence for decreased structurally determined preglomerular resistance in the young spontaneously hypertensive rat after 4 weeks of renal denervation. J Hypertens. 1997; 15: 1187-1195.

8. Anderson WP, Kett MM, Evans RG, Alcorn D. Pre-glomerular structural changes in the renal vasculature in hypertension. Blood Press. 1995; 4 (Suppl 2): 74-80.

9. Yu SM, Tsai SY, Guh JH, Ko FN, Teng CM, Ou JT. Mechanism of catecholamine-induced proliferation of vascular smooth muscle cells. Circulation. 1996; 
94: 547-554.

10. Bobik A, Dilley R, Kanellakis P. Sympatho-adrenal mechanisms regulating cardiovascular hypertrophy in primary hypertension: a role for rilmenidine? J Hypertens. 1998; 16 (suppl 3): S51-S55.

11. DiBona GF, Jones SY. Analysis of renal sympathetic nerve responses to stress. Hypertension. 1995; 25: 531-538.

12. Head RJ. Hypernoradrenergic innervation: its relationship to functional and hyperplastic changes in the vasculature of the spontaneously hypertensive rat. Blood Vessels. 1989; 26: 1-20.

13. Levi-Montalcini R, Dal Toso R, della Valle F, Skaper SD, Leon A. Update of the NGF saga. J Neurol Sci. 1995; 130: 119-127.

14. Rush RA, Chie E, Liu D, Tafreshi A, Zettler C, Zhou XF. Neurotrophic factors are required by mature sympathetic neurons for survival, transmission and connectivity. Clin Exp Pharmacol Physiol. 1997; 24: 549-555.

15. Falckh PH, Harkin LA, Head RJ. Nerve growth factor mRNA content parallels altered sympathetic innervation in the spontaneously hypertensive rat. Clin Exp Pharmacol Physiol. 1992; 19: 541-545.

16. Bonofiglio R, Antonucci MT, Papalia $\mathrm{T}$ et al. Nerve growth factor (NGF) and 
NGF-receptor expression in diseased human kidneys. J Nephrol. 2007; 20: 186-195.

17. Zhou S, Chen LS, Miyauchi Y et al. Mechanisms of cardiac nerve sprouting after myocardial infarction in dogs. Circ Res. 2004; 95: 76-83.

18. Zettler C, Rush RA. Elevated concentrations of nerve growth factor in heart and mesenteric arteries of spontaneously hypertensive rats. Brain Res.1993; 614: 15-20.

19. Ueyama T, Hamada M, Hano T, Nishio I, Masuyama Y, Furukawa S. Increased nerve growth factor levels in spontaneously hypertensive rats. J Hypertens. 1992; 10: 215-219.

20. Charchar FJ, Kapuscinski MK, Harrap SB. Nerve growth factor gene locus explains elevated renal nerve growth factor mRNA in young spontaneously hypertensive rats. Hypertension. 1998; 32: 705-709.

21. Brock JA, Van Helden DF, Dosen P, Rush RA. Prevention of high blood pressure by reducing sympathetic innervation in the spontaneously hypertensive rat. J Auton Nerv Syst. 1996; 61: 97-102.

22. Folkow B, Hallbäck M, Lundgren Y, Weiss L. The effects of "immunosympathectomy" on blood pressure and vascular "reactivity" in normal and spontaneously hypertensive rats. Acta Physiol Scand. 1972; 84: 512-523.

23. Korner P, Bobik A, Oddie C, Friberg P. Sympathoadrenal system is critical for 
structural changes in genetic hypertension. Hypertension. 1993; 22: 243-252.

24. Lee RM, Borkowski KR, Leenen FH, Tsoporis J, Coughlin M. Combined effect of neonatal sympathectomy and adrenal demedullation on blood pressure and vascular changes in spontaneously hypertensive rats. Circ Res. 1991; 69: 714-721.

25. Cutilletta AF, Oparil S. Hemodynamic response to vascular expansion following immunosympathectomy in spontaneously hypertensive rats. Hypertension. 1980; 2: 304-310.

26. Zettler C, Head RJ, Rush RA. Chronic nerve growth factor treatment of normotensive rats. Brain Res. 1991; 538: 251-262.

27. Caporali A, Emanueli C. Cardiovascular actions of neurotrophins. Physiol Rev. 2009; 89: 279-308.

28. Hristova MG. Metabolic syndrome-from the neurotrophic hypothesis to a theory. Med Hypotheses. 2013; 81: 627-634.

29. Aloe L, Rocco ML, Bianchi P, Manni L. Nerve growth factor: from the early discoveries to the potential clinical use. J Transl Med. 2012; 10: 239.

30. Mulvany MJ. Vascular remodeling of resistance vessels: can we define this? Cardiovasc Res. 1999; 41: 9-13. 
31. Gibbons GH, Dzau VJ. The emerging concept of vascular remodeling. $N$ Engl $J$

Med. 1994; 330: 1431-1438.

32. Lohmeier TE, Hildebrandt DA, Warren S, May PJ, Cunningham JT. Recent insights into the interactions between the baroreflex and the kidneys in hypertension. Am J Physiol Regul Integr Comp Physiol. 2005; 288: R828-836.

33. Branchi I, Francia N, Alleva E. Epigenetic control of neurobehavioural plasticity: the role of neurotrophins. Behav Pharmacol. 2004; 15: 353-362.

34. Clark CG, Hasser EM, Kunze DL, Katz DM, Kline DD. Endogenous brain-derived neurotrophic factor in the nucleus tractus solitarius tonically regulates synaptic and autonomic function. J Neurosci. 2011; 31: 12318-12329.

35. Tomoda F, Takata M, Kinuno H, Tomita S, Yasumoto K, Inoue H. Renal structural properties in prehypertensive Dahl salt-sensitive rats. Hypertension. 2000; 36: 68-72.

36. Barajas L, Powers K. Monoaminergic innervation of the rat kidney: a quantitative study. Am J Physiol. 1990; 259: F503-511.

37. Esler M, Lambert G, Brunner-La Rocca HP, Vaddadi G, Kaye D. Sympathetic nerve activity and neurotransmitter release in humans: translation from pathophysiology into clinical practice. Acta Physiol Scand. 2003; 177: 275-284. 
38. Lee TM, Chen CC, Chang NC. Cardiac sympathetic hyperinnervation in deoxycorticosterone acetate-salt hypertensive rats. Clin Sci. 2012; 123: 445-457.

39. Uno K, Takarada $T$, Takarada-Iemata $M$ et al. Negative regulation of osteoblastogenesis through downregulation of runt-related transcription factor-2 in osteoblastic MC3T3-E1 cells with stable overexpression of the cystine/glutamate antiporter xCT subunit. J Cell Physiol. 2011; 226: 2953-2964.

40. Laborda J. 36B4 cDNA used as an estradiol-independent mRNA control is the cDNA for human acidic ribosomal phosphoprotein PO. Nucleic Acids Res. 1991; 19: 3998.

\section{Figure legends}

Figure 1 Relationships between inflow volume and arterial distending pressure in spontaneously hypertensive rats (SHR: d-f) and Wistar-Kyoto (WKY) rats (a-c). Results are shown as individual lines in rats treated with vehicle (a, d) or antiserum against nerve growth factor (anti-NGF) (b, e). Averaged lines of the relationship in rats treated with vehicle (dashed lines) or anti-NGF (solid lines) were also shown (c, f). In the rats treated with vehicle, the gradient of the flow-pressure relationship was significantly greater in SHR than in WKY $(P<0.001)$. The gradient of the line was less in SHR treated with anti-NGF compared with SHR treated with vehicle $(P<0.001)$, although it 
did not differ significantly between WKY rats treated with anti-NGF and WKY rats treated with vehicle $(P=0.19)(P=0.009$ for strain $\times$ treatment interaction $)$. As a result, the gradient of line did not differ significantly between SHR treated with anti-NGF and WKY treated with vehicle $(P=0.47)$. Differences between the strains and differences between the treatments were determined using one-way analysis of variance (ANOVA). Comparisons of the changes induced by anti-NGF between the 2 strains were tested by two-way ANOVA.

Figure 2 Relationships between arterial distending pressure and glomerular filtration rate (GFR) in spontaneously hypertensive rats (SHR) (d-f) and Wistar-Kyoto (WKY) rats (a-c). Results are shown as individual lines in rats treated with vehicle (a, d) or antiserum against nerve growth factor (anti-NGF) (b, e). Averaged lines of the relationship in rats treated with vehicle (dashed lines) or anti-NGF (solid lines) were also shown (c, f). In the rats treated with vehicle, the $\mathrm{X}$ intercept of the pressure-GFR relationship was significantly higher in SHR than in WKY rats $(P<0.001)$, although the gradient of the line did not differ significantly between the two strains $(P=0.15)$. The gradient of the line was greater in SHR treated with anti-NGF than in SHR treated with vehicle $(P=0.003)$, although it did not differ significantly between WKY rats treated with anti-NGF and WKY rats treated with vehicle $(P=0.30)(P=0.036$ for strain $\times$ 
treatment interaction). As a result, the gradient of the line was greater in SHR treated with anti-NGF than in WKY rats treated with vehicle $(P=0.004)$. Contrary, anti-NGF did not affect the X-intercept of the line in either strain. Differences between the strains and differences between the treatments were determined using one-way analysis of variance (ANOVA). Comparisons of the changes induced by anti-NGF between the 2 strains were tested by two-way ANOVA.

Figure 3 Morphological characteristics of preglomerular vessels in maximally vasodilated kidneys at 10 weeks of age in spontaneously hypertensive rats (SHR) and Wistar-Kyoto rats (WKY) treated with antiserum against nerve growth factor (anti-NGF) or vehicle at 3 weeks of age. (c) and (d) show interlobular artery of SHR treated with vehicle and anti-NGF, respectively. (a) and (b) show interlobular artery of WKY treated with vehicle and anti-NGF, respectively. See text for details. Original photography taken by $\times 400$, each bar shows $50 \mu \mathrm{m}$. 